\title{
The TLC: A Novel Auditory Nucleus of the Mammalian Brain
}

\author{
Enrique Saldaña, ${ }^{1,2}$ Antonio Viñuela, ${ }^{1,2}$ Allen F. Marshall, ${ }^{3}$ Douglas C. Fitzpatrick, ${ }^{3}$ and M.-Auxiliadora Aparicio ${ }^{1,2}$ \\ ${ }^{1}$ Laboratory for the Neurobiology of Hearing, Department of Cell Biology and Pathology, Medical School, University of Salamanca, 37007-Salamanca, Spain, \\ ${ }^{2}$ Neuroscience Institute of Castilla y León (INCyL), University of Salamanca, 37007-Salamanca, Spain, and ${ }^{3}$ Department of Otolaryngology-Head and Neck \\ Surgery, University of North Carolina School of Medicine, Chapel Hill, North Carolina 27599-7070
}

We have identified a novel nucleus of the mammalian brain and termed it the tectal longitudinal column (TLC). Basic histologic stains, tract-tracing techniques and three-dimensional reconstructions reveal that the rat TLC is a narrow, elongated structure spanning the midbrain tectum longitudinally. This paired nucleus is located close to the midline, immediately dorsal to the periaqueductal gray matter. It occupies what has traditionally been considered the most medial region of the deep superior colliculus and the most medial region of the inferior colliculus. The TLC differs from the neighboring nuclei of the superior and inferior colliculi and the periaqueductal gray by its distinct connections and cytoarchitecture. Extracellular electrophysiological recordings show that TLC neurons respond to auditory stimuli with physiologic properties that differ from those of neurons in the inferior or superior colliculi. We have identified the TLC in rodents, lagomorphs, carnivores, nonhuman primates, and humans, which indicates that the nucleus is conserved across mammals. The discovery of the TLC reveals an unexpected level of longitudinal organization in the mammalian tectum and raises questions as to the participation of this mesencephalic region in essential, yet completely unexplored, aspects of multisensory and/or sensorimotor integration.

Key words: superior colliculus; inferior colliculus; auditory; periaqueductal gray; midbrain tectum; multisensory; sensorimotor; tracttracing; electrophysiology; 3D reconstruction; rat; human

\section{Introduction}

In mammals, the roof of the midbrain (the mesencephalic tectum) consists primarily of the paired superior and inferior colliculi, which are visible on the dorsal surface of the brainstem as four symmetrically arranged bumps, the corpora quadrigemina. The superior colliculus (SC) is a complex, multilayered structure (Huerta and Harting, 1984; May, 2005). Its superficial layers receive visual information, whereas its deep layers receive convergent visual, auditory and somatosensory information, and are involved in the genesis of motor responses, including gaze control and head and pinna postural adjustments (Sparks and Nelson, 1987; King, 2004). The inferior colliculus (IC) occupies a

\footnotetext{
Received April 26, 2007; revised 0ct. 10, 2007; accepted 0ct. 11, 2007.

This work was supported by the Spanish Ministry of Science and Technology Grants BFI2000/1358 and BFU200405909 (E.S.), Junta de Castilla y León Grants SA097/01 and SA007C05 (E.S.), National Institutes of Health (NIH) Grant DC04398 (D.C.F.), and the NIH Short Term Research Training Grant and the University of North Carolina Medical Alumni Association Endowment Fund (A.F.M.). A.V. was supported by Predoctoral Fellowship BEFI00/9418 from the Instituto de la Salud Carlos III and M.-A.A. was supported by Predoctoral Fellowship FPU AP2002/3330 from the Spanish Ministry of Education. We thank Albert S. Berrebi, Fernán Jaramillo, Enrico Mugnaini, Kirsten K. Osen, and Nobuo Suga for helpful discussions and comments on this manuscript, Manuel $S$. Malmierca for assistance with the 3 reconstructions, and Enrique A. López-Poveda and Ana Alves-Pinto for assistance with the statistical analysis. The human brainstems were made available by Ángel Santos-Briz and María-Dolores Ludeña, the sections of the crabeating monkey brainstems by Javier Cervera-Paz and Manuel Manrique, and those of ferret brainstems by Francisco Clascá. E.S. discovered the TLC, designed all morphological experiments, supervised the project, and wrote this manuscript. A.V. did most of the tract-tracing experiments and the 3 D reconstructions. A.F.M. and D.C.F. performed the electrophysiological recordings. M.-A.A. studied the comparative anatomy of the TLC and performed the morphometric and stereological analyses.

Correspondence should be addressed to Dr. Enrique Saldaña, Laboratorio de Neurobiología de la Audición, Departamento de Biología Celular y Patología, Facultad de Medicina, Universidad de Salamanca, 37007 Salamanca, Spain. E-mail: saldana@usal.es.

DOl:10.1523/JNEUROSCI.1892-07.2007

Copyright $\odot 2007$ Society for Neuroscience $\quad 0270-6474 / 07 / 2713108-09 \$ 15.00 / 0$
}

pivotal position in both ascending and descending auditory pathways (Aitkin, 1986). The left and right SC are interconnected by numerous axons that travel in the rostral half of the commissure of the SC (CoSC) (Edwards, 1977; Olivier et al., 2000); likewise, the left and right IC are interconnected through the commissure of the IC (CoIC) (for review, see Saldaña and Merchán, 2005).

Whereas the main portions of the SC and IC have been extensively analyzed anatomically and functionally (Hall and Moschovakis, 2004; Winer and Schreiner, 2005), our studies focused on a region of the midbrain tectum that has not been analyzed in detail previously, namely the paramedian region that includes the medialmost portions of the deep SC and the IC. Here, we report that this medial territory of the tectum contains a previously unidentified nucleus of the rat's midbrain. We refer to it as the tectal longitudinal column (TLC) because of its shape and position. We provide evidence based on cytoarchitecture, fiber connections and basic electrophysiology that the TLC is a distinct nucleus of the rat brain. Furthermore, we demonstrate that the TLC is present in a large variety of mammals, including humans.

\section{Materials and Methods}

Experimental animals. For the neuroanatomical studies, Sprague Dawley rats of either sex (body weight, 190-210 g) were cared for and used in compliance with European Union regulations concerning the use of animals in biomedical research, and the experimental procedures were approved and supervised by the Animal Care and Use Committee of the University of Salamanca. For the surgical procedures, including the transcardial perfusion of fixatives, the animals were deeply anesthetized with a mixture of ketamine $\mathrm{HCl}(80 \mathrm{mg} / \mathrm{kg}$ body weight $)$ and xylazine (6 $\mathrm{mg} / \mathrm{kg}$ body weight) administered intramuscularly.

The electrophysiological recordings were obtained from Sprague 
Dawley and Long-Evans rats, and all the experimental procedures conformed to the National Institutes of Health guidelines and protocols approved by the Animal Care and Use Committee of The University of North Carolina. Throughout the recording sessions, the rats were deeply anesthetized with urethane $(25 \%$ in saline, $1.5 \mathrm{~g} / \mathrm{kg})$ administered intraperitoneally.

Human nervous tissue. We examined sections of the brainstem of several adults, both males and females, who died from non-neurological causes, and whose brain was judged normal both macroscopically and microscopically. The time from death to the immersion in fixative (4\% formaldehyde prepared from commercial Formalin) was 3-12 h. After several weeks in fixative, the brainstems were blocked, cryoprotected with sucrose, and sectioned on a freezing microtome in a plane transverse to the longitudinal axis of the mesencephalon. All procedures involving human material were approved and supervised by the Bioethics Committee of the Clinical Hospital of the University of Salamanca.

Cytoarchitectural analysis. We studied 40 - to $60-\mu \mathrm{m}$-thick frozen sections or $15-\mu \mathrm{m}$-thick paraffin-embedded sections of the rat, mouse, hamster, gerbil, chinchilla, guinea pig, rabbit, cat and human midbrain tectum stained with either $0.25-1 \%$ cresyl violet or with the Giemsa method (Íñiguez et al., 1985). This material was available from the histological collection of the Laboratory for the Neurobiology of Hearing of the University of Salamanca.

We also used semithin sections of the rat TLC stained with toluidine blue. To produce these, rats were deeply anesthetized and perfused with fixative containing $2.5 \%$ glutaraldehyde and $2 \%$ formaldehyde (prepared from freshly depolymerized paraformaldehyde). Vibratome sections ( $80 \mu \mathrm{m}$ thick) were postfixed with $1 \% \mathrm{OsO}_{4}$, stained with uranyl acetate, dehydrated, cleared with propylene oxide and flat-embedded in Epon 812 resin. Fragments of sections containing the TLC were cut out, re-embedded into blank resin blocks, and sectioned with an ultramicrotome at a thickness of $1-2 \mu \mathrm{m}$. These semithin sections were finally stained with $1 \%$ toluidine blue.

Sections of the brainstem of ferrets and monkeys were obtained from the histological collections of other institutions listed in the acknowledgments. Such sections had been processed by routine neurohistological methods, including Nissl staining with cresyl violet or thionin.

Unbiased stereological estimates of neuron numbers. We selected for study three series of coronal sections from our collection of rat brains stained by the Giemsa method (Íñiguez et al., 1985). We chose Giemsastained material because this polychromatic technique provides a clear distinction between purple-stained neurons and light blue-stained glial and endothelial cells. This was particularly useful given the small size of most TLC neurons.

We followed procedures described previously (Kulesza et al., 2002). Stereo Investigator software (MBF Bioscience, Williston, VT) was used to implement the optical fractionator protocol, which encompasses the optical dissector and fractionator tools (Gundersen, 1988, Gundersen et al., 1988). To minimize the coefficient of error, we counted from every fourth section and set the parameters of the optical fractionator so that the sample consisted of at least 250 neurons for each TLC. This was achieved by sampling from approximately fifteen $400 \mu \mathrm{m}^{2}$ counting frames per section.

Tract-tracing with FluoroGold. Glass micropipettes loaded with the retrograde tracer FluoroGold (Fluorochrome, Denver, CO; $4 \%$ in saline) were stereotaxically placed into the superior olivary complex of deeply anesthetized rats $(n=11)$ and the tracer was delivered iontophoretically by passing a pulsed $5 \mu \mathrm{A}$ positive direct current ( $7 \mathrm{~s}$ on/ $7 \mathrm{~s}$ off) for 5-15 min. The current was then stopped and the pipette left in place for an additional 15-20 min before withdrawal to prevent leakage of the tracer along the injection tract.

After 5-10 d of survival, the rats were anesthetized deeply and their brains fixed by transcardial perfusion of buffered $4 \%$ formaldehyde (prepared from freshly depolymerized paraformaldehyde). The brains were cut coronally $(n=6)$ or parasagittally $(n=5)$ on a freezing microtome at a thickness of $40 \mu \mathrm{m}$.

Initially, we assessed the location of the injection site and labeled neurons by inspecting representative fresh sections on a Leica DMRB microscope under epifluorescence illumination. In selected cases the Fluoro-
Gold was rendered permanently visible by immunocytochemistry on free-floating sections, using a rabbit anti-FluoroGold primary antiserum (1:4000; Millipore, Temecula, CA) followed by biotinylated anti-rabbit immunoglobulin G raised in goat (1:50; Vector Laboratories, Burlingame, CA), and then by incubation in the avidin-biotin-peroxidase complex (Vectastain; Vector Laboratories) and standard histochemistry for peroxidase, with or without heavy-metal intensification.

Three-dimensional reconstructions. In selected cases, we generated three-dimensional (3D) reconstructions of the location of the TLC neurons labeled with FluoroGold. Every other coronal section was drawn at $25 \times$ with the aid of a drawing tube attached to a Leica DMRB microscope. Each drawing included: (1) the outline of the section and the midline, (2) the outline of the TLC and adjacent anatomical structures, (3) the location of each labeled TLC neuron, and (4) the location of major blood vessels and other fiduciary marks useful to align the drawings of consecutive sections. The drawings were then aligned and digitized with a GTCO CalComp (Columbia, MD) graphic pad using Microtrace software (Leergaard and Bjaalie, 1995). Three-dimensional images were finally produced with a Silicon Graphics (Sunnyvale, CA) workstation.

Morphometry. For comparison of neuronal size across structures, we used a $100 \times$ oil-immersion objective (numerical aperture, 1.4) to measure the maximum diameter of the neuronal cell bodies in semithin, coronal sections through the central third of the rat TLC. In each section, we measured all neurons with visible nucleoli located in the TLC, dorsomedial column of the periaqueductal gray matter (PAGdm), medial SC and "dorsal column." Neurons in the PAGdm were limited to those located within $200 \mu \mathrm{m}$ of the ventral border of the TLC, and SC neurons to those within $200 \mu \mathrm{m}$ of the lateral border of the TLC.

For comparison of density across structures, relative neuronal density was determined using $15-\mu \mathrm{m}$-thick paraffin-embedded sections of the central third of the rat TLC stained by the Giemsa method. Using a 100X oil-immersion objective (numerical aperture, 1.4), for each section we counted the neurons included in two square areas of $2500 \mu \mathrm{m}^{2}$ randomly placed in each one of the structures analyzed: TLC, PAGdm, medial SC, and "dorsal column". The diameter and packing density of TLC neurons were compared with those of the PAGdm, medial SC and dorsal column using Student's $t$ test for comparisons of two groups.

Electrophysiological recordings. We recorded the activity of single neurons and small clusters of neurons in the TLC of albino Sprague Dawley rats and pigmented Long-Evan rats anesthetized with urethane. Glassinsulated tungsten electrodes $(10-20 \mathrm{M} \Omega$ impedance at $1 \mathrm{kHz})$ were inserted into the brain vertically, $\sim 200 \mu \mathrm{m}$ off the midline, so that they traversed the prominent superior sagittal sinus. This maneuver resulted in hardly any bleeding while the electrode was advanced into the brain or left in place for the recordings, and only moderate bleeding after withdrawal of the electrode. We aimed preferentially at the central rostrocaudal third of the TLC, which is not crossed by fascicles of commissural fibers. In each penetration, we tested for responsiveness to light with a static flashlight or laser pointer in the darkened sound-attenuated chamber, and for responsiveness to sound with a search stimulus consisting of wideband noise at 50-80 dB SPL. Acoustic stimuli were delivered through Beyer DT-48 earphones coupled to hollow sound tubes that matched the diameter of the ear canal, with a cone of hard foam $5 \mathrm{~mm}$ from end of the sound tube to form a sealed system. Stimuli at each ear were calibrated in amplitude and phase through a probe tube that ran within the sound tube. The stimuli were generated using System II components from Tucker-Davis Technologies (Alachua, FL), and consisted of wideband noise and tones delivered independently to each ear. In most cases, we confirmed the location of the recording site in the TLC by identifying in histological sections an electrolytic lesion made at the end of the recording session.

\section{Results}

\section{Size and position of the rat TLC}

The rat TLC is a long and narrow nucleus that spans the midbrain tectum longitudinally, very close to the midline and dorsal to the dorsomedial column of PAGdm (Fig. 1). It extends from the caudal end of the CoIC to the rostral part of the CoSC (Fig. 1A), 

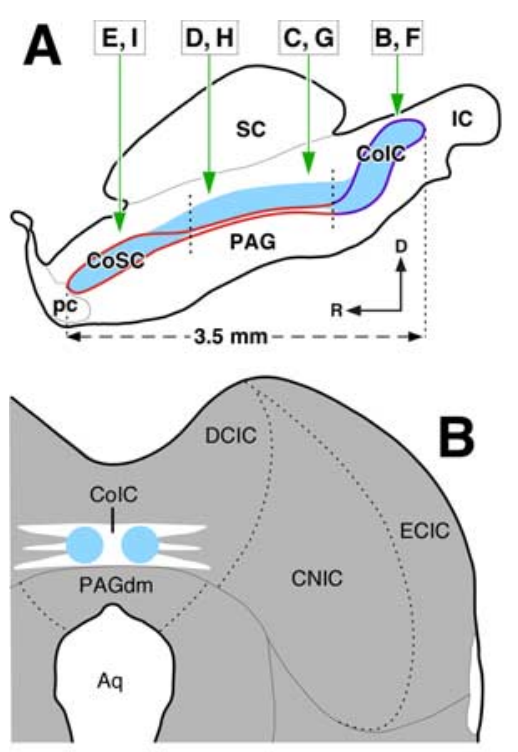

I.A. $0.8 \mathrm{~mm}$

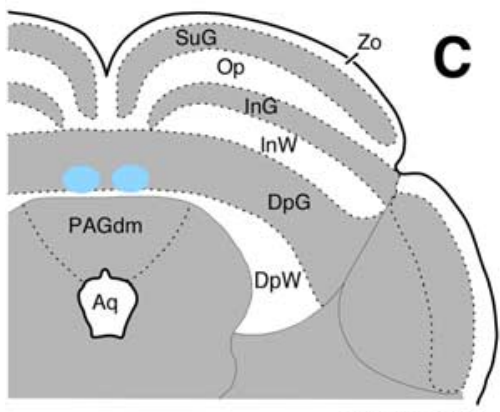

I.A. $1.6 \mathrm{~mm}$

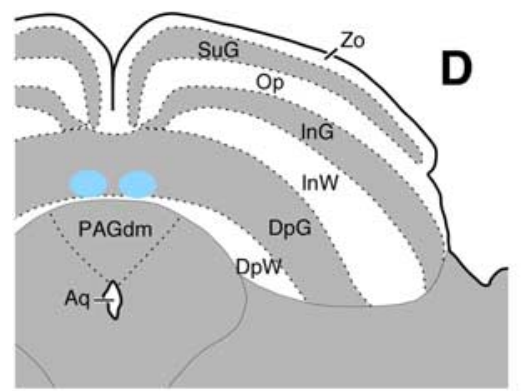

I.A. $2.5 \mathrm{~mm}$

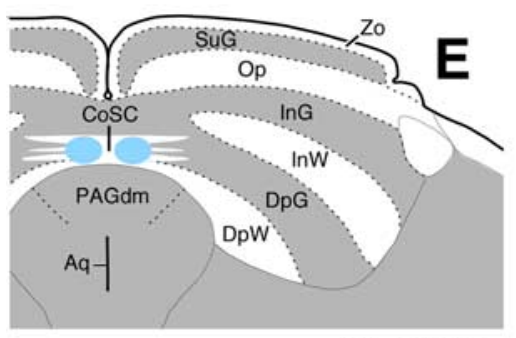

I.A. $3.3 \mathrm{~mm}$
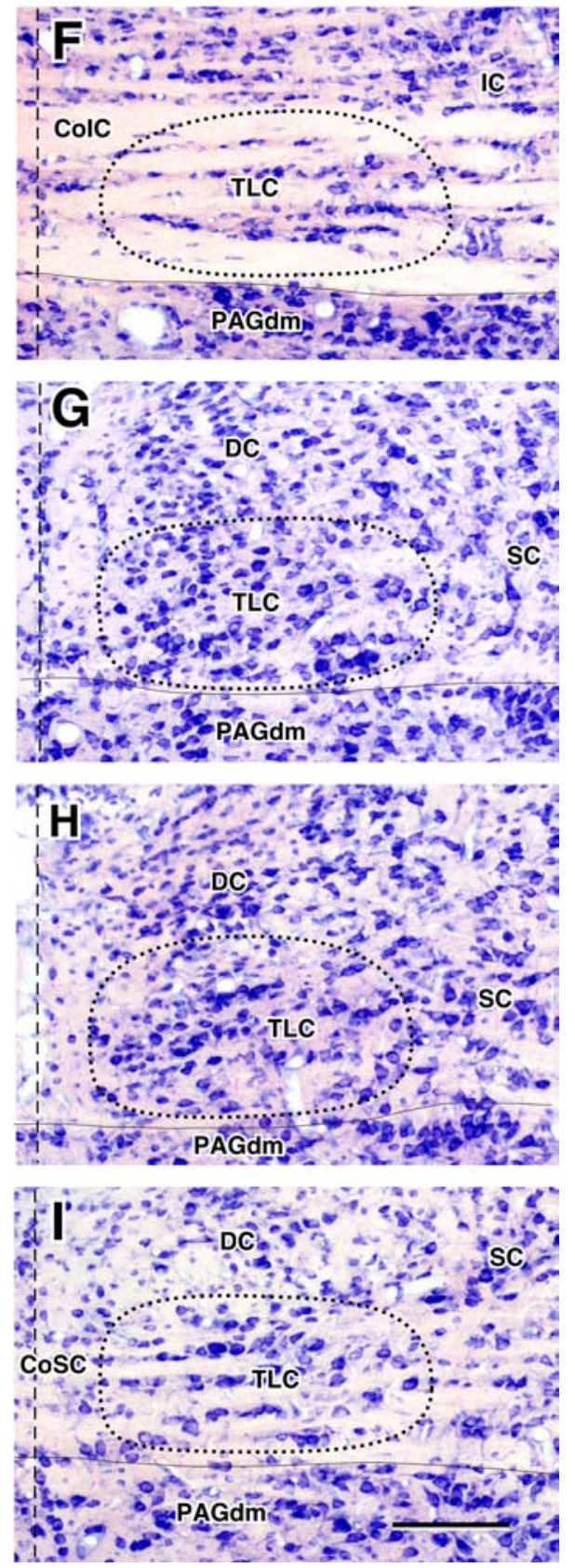

Figure 1. Size and position of the rat TLC. $A$, Schematic drawing of a very medial parasagittal section of the rat midbrain tectum $200 \mu \mathrm{m}$ lateral to the midsagittal plane, showing the relationships between the TLC (blue), the tectal commissures and the PAG. The vertical dotted lines indicate the limits between the caudal, the central and the rostral TLC. The caudal third of the TLC is crossed by the ColC (outlined in violet). The rostral third of the nucleus is traversed by the enlarged, rostral half of the CoSC (outlined in red). The central third of the TLC lies above the narrow, caudal half of the COSC. The vertical green arrows indicate the rostrocaudal level of the coronal sections shown in $\boldsymbol{B}-\boldsymbol{I} . \boldsymbol{B}-\boldsymbol{E}$, Schematic drawings of four idealized coronal sections of the rat midbrain tectum taken at the level of the rostral IC (B), and the caudal ( $\boldsymbol{C}$, central (D) and rostral (E) SC. The number at the bottom of each schematic indicates the distance in millimeters between the depicted plane and the interaural coronal plane (I.A.). The TLC has been represented in blue. Scale bar, $1 \mathrm{~mm}$. $\boldsymbol{F}$-I, Digital micrographs of four $15-\mu \mathrm{m}$-thick paraffin-embedded coronal sections of the rat TLC stained by the Giemsa method. Each section comes from a level that matches the one depicted in the corresponding and is slightly tilted from caudal and dorsal to rostral and ventral. Its length is $\sim 3.5$ $\mathrm{mm}$, and its thickness is $\sim 250 \mu \mathrm{m}$ in the dorsoventral dimension and $350 \mu \mathrm{m}$ in the mediolateral dimension. In parasagittal sections the TLC possesses a quasisigmoid shape (Fig. 1A), whereas in coronal sections it appears as a round to oval structure that occupies the angle defined by the midline and the dorsal border of the PAG (Fig. $1 B-I$ ). The distance between the medial border of the TLC and the midline $(50-100 \mu \mathrm{m})$ is remarkably constant throughout the length of the nucleus. The number of TLC neurons on each side is $\sim 11,500$ (mean, 11,523.16 $\pm 549.34 \mathrm{SD}$, $n=6$ ), as estimated using methods of unbiased stereology, and it does not differ between the two sides ( $t$ test, $p=0.662$; left side, $11,315.66 \pm 797.55 \mathrm{SD}, n=3$; right side, 11,730.66 $\pm 374.26 \mathrm{SD}, n=3$ ).

\section{The TLC is hodologically distinct}

The TLC is best revealed by tract-tracing experiments (Faye-Lund, 1986). We injected the sensitive retrograde tracer, FluoroGold, into the superior olivary complex of adult rats (Fig. $2 A, A^{\prime}$ ). FluoroGold provides excellent filling of the cell bodies and dendrites of the labeled neurons. In addition to the expected labeling in all nuclei known to innervate the superior olivary complex (reviewed by Thompson and Schofield, 2000), abundant neurons were labeled in the TLC ipsilateral to the injection site (Fig. $2 B-E$ ). In the caudal and rostral TLC, labeled neurons occupied the spaces between the fascicles of commissural fibers (Fig. 2B,E), whereas in the central TLC the neurons were more closely packed (Fig. 2C,D). This dense retrograde labeling allowed the unequivocal distinction of the TLC from the adjacent medial IC, medial SC and PAGdm (Fig. 2). In the contralateral TLC, labeled neurons were scarce (Fig. 2).

In parasagittal sections, the FluoroGold-labeled TLC neurons formed a long and well defined column that spanned the midbrain tectum longitudi-

scheme on the left. The vertical dashed lines indicate the midline. Because of the commissural fascicles that cross the TLC, the neuronal density is lower in the caudal $(\boldsymbol{F})$ and the rostral $(\boldsymbol{I})$ thirds than in the central third $(\boldsymbol{G}, \boldsymbol{H})$ of the nucleus. Scale bar, $100 \mu \mathrm{m}$, uncorrected for shrinkage. CNIC, Central nucleus; DCIC, dorsal cortex; ECIC, external cortex; DpG, deep gray; $D p W$, deep white; InG, intermediate gray; InW, intermediate white; Op, stratum opticum; SuG, superficial gray; Zo, stratum zonale; Aq, cerebral aqueduct; PAGdm, dorsomedial column of the periaqueductal gray matter; $\mathrm{pC}$, posterior commissure; DC, dorsal column. 

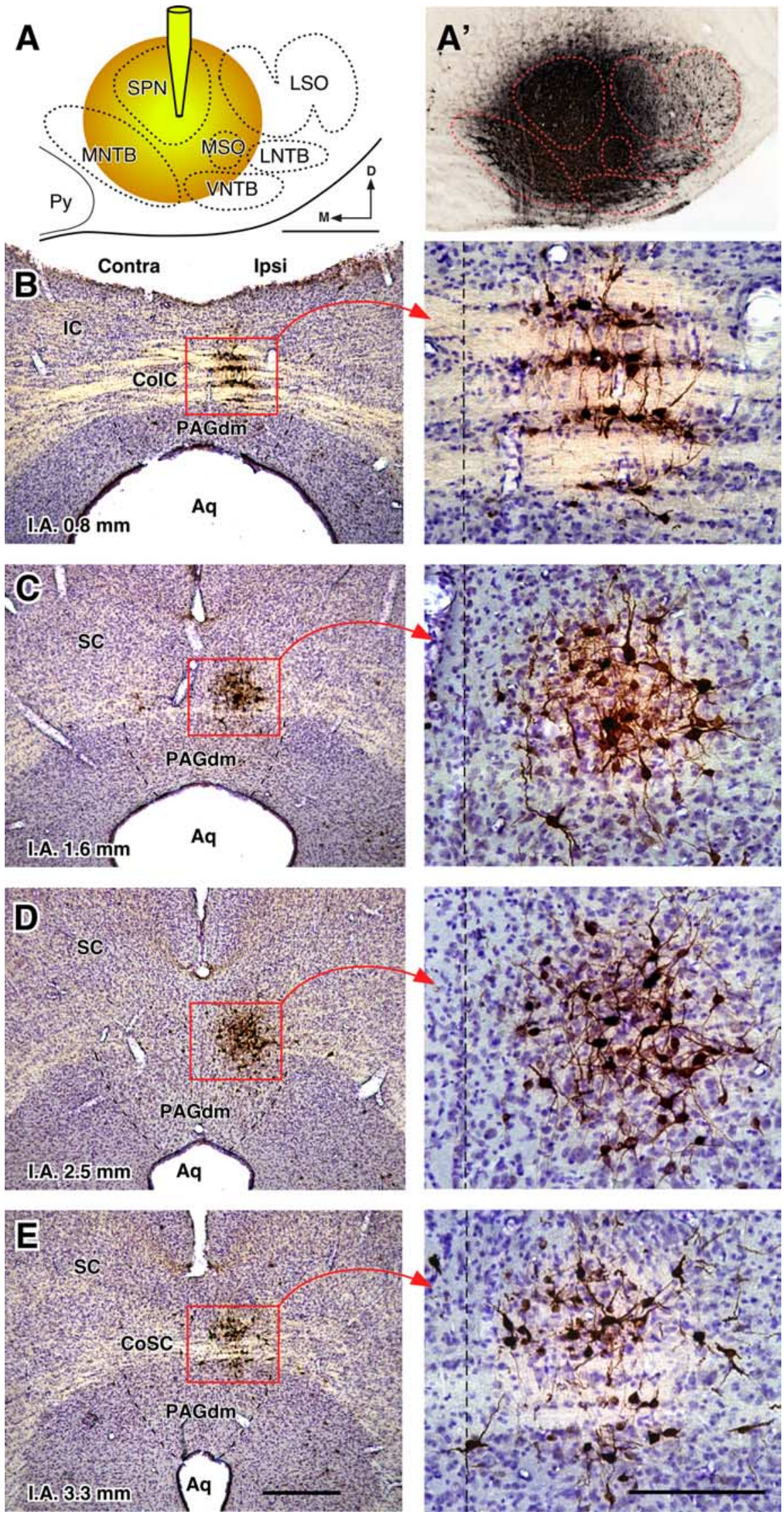

Figure 2. The TLC is labeled following injections of a retrograde tracer into the superior olivary complex. $\boldsymbol{A}, \boldsymbol{A}^{\prime}$, Schematic drawing $(\boldsymbol{A})$ and digital micrograph $\left(\boldsymbol{A}^{\prime}\right)$ of an injection site of FluoroGold into the right superior olivary complex of the rat. The injection site included most superior olivary complex nuclei and the ventral margin of the overlying reticular formation, and spared the rostrally located pontine nuclei, the medially located pyramidal tract and the caudally located facial motor nucleus. $\boldsymbol{B}-\boldsymbol{E}$, Pairs nally from the CoIC through the CoSC (Fig. 3). No labeled neurons were found in the posterior commissure.

The cylindrical, opened sigmoid shape of the TLC is best appreciated with $3 \mathrm{D}$ reconstructions of the position of TLC neurons labeled after injections of FluoroGold restricted to the ipsilateral superior olivary complex (Fig. 4A). The 3D reconstructions also emphasize the remarkable parallelism between the rostrocaudal axis of the TLC and the midline (Fig. $4 B$ ).

As shown in Figure 2, $A$ and $A^{\prime}$, our injection sites were relatively large and the tracer spread into various nuclei of the superior olivary complex. Nevertheless, a comparison between cases suggests that a higher number of retrogradely labeled TLC neurons was associated with a larger involvement of the superior paraolivary nucleus within the injection site. Moreover, the fact that numerous TLC neurons were labeled in cases in which the injection site was completely restricted to the superior olivary complex indicates that this labeling was not caused by spread of the tracer outside the complex. Retrogradely labeled neurons were observed in the PAGdm in cases in which the tracer spread into the reticular formation overlying the superior olivary complex.

The TLC is cytoarchitecturally distinct The identification of the TLC by experimental neuroanatomy engendered the need for a cytoarchitectural study. The caudal third of the TLC is separated from the rest of the IC by a visible gap and is crossed by fascicles of axons of the CoIC (Fig. 1B,F). The central third of the TLC stands out from the neighboring medial SC because of its significantly higher neuronal density (Figs. 1G, $\mathrm{H}, 5 \mathrm{~A}$ ). It is not crossed by conspicuous commissural fascicles, as it lies immediately above the caudal half of the CoSC. At this level, the thin

of digital micrographs of four coronal sections of the rat brainstem tectum showing $\mathrm{TLC}$ neurons retrogradely labeled in the case whose injection site is depicted in $\boldsymbol{A}$ and $\boldsymbol{A}^{\prime}$. The number at the bottom of each left micrograph indicates the distance in millimeters between the depicted plane and the interaural coronal plane (I.A.). In each pair the picture on the right shows at higher magnification the area boxed in red. Note that labeled neurons are abundant and widespread within the right $\mathrm{TLC}$, ipsilateral to the injection site, but are virtually absent in the surrounding nuclei, as well as in the left TLC. The midline is indicated by the vertical dashed line. Scale bars: $A, A^{\prime}, 0.5 \mathrm{~mm}$; $\boldsymbol{E}$, left, $1 \mathrm{~mm}$; right, $200 \mu \mathrm{m}$. Aq, Cerebral aqueduct; LNTB, lateral nucleus of the trapezoid body; $\mathrm{LSO}$, lateral superior olive; MNTB, medial nucleus of the trapezoid body; MSO, medial superior olive; Py, pyramidal tract; SPN, superior paraolivary nucleus; VNTB, ventral nucleus of the trapezoid body. 


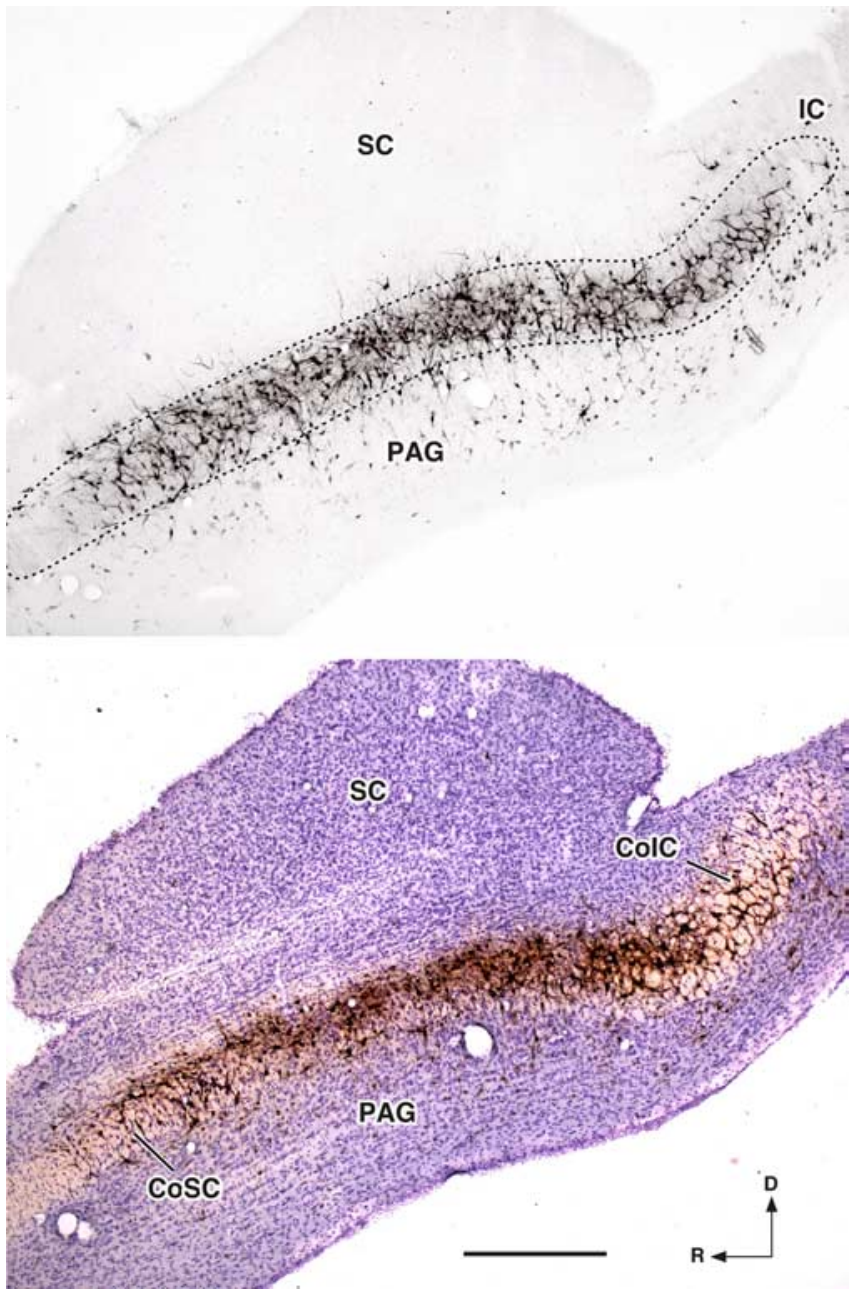

Figure 3. The full length of the rat $\mathrm{TLC}$ is revealed in parasagittal sections. Digital photomicrographs of two adjacent parasagittal sections through the medial midbrain tectum from a case in which the retrograde tracer FluoroGold was injected into the ipsilateral superior olivary complex. Labeled neurons are visible throughout the rostrocaudal extent of the TLC. In the section at the top, the tracer was detected immunocytochemically and revealed in black with a nickel-enhanced peroxidase reaction. The section at the bottom was subsequently counterstained by the Nissl method. Scale bar, $0.5 \mathrm{~mm}$. R, Rostral; D, dorsal.

fiber fascicles of the CoSC wrap around the PAG dorsally and create a sharp natural border between the TLC and the PAG (Fig. $1 C, D, G, H)$. The rostral third of the TLC is crossed by the enlarged, rostral half of the CoSC (Fig. $1 E$ ). Accordingly, in sections through the rostral SC, the TLC neurons are distributed in horizontal rows (Fig. 1I).

The cytoarchitecture of the TLC is distinct from that of the neighboring territories (Fig. 5). TLC neurons are relatively homogeneous. Most of them have small cell bodies, with their main axis oriented rostrocaudally. Consequently, TLC somata appear elongated (average maximum diameter, $16 \mu \mathrm{m}$ ) and are distributed parallel to each other in horizontal sections (Fig. $5 A$ ), whereas in coronal sections they tend to be rounded (Fig. 5E) (average maximum diameter, $13 \mu \mathrm{m}$ ). The cell nucleus occupies most of the soma and shows several indentations (Fig. 5E). Scattered throughout the TLC are a few medium-sized neurons (average cell body diameter, $25 \mu \mathrm{m}$ in horizontal sections and $18 \mu \mathrm{m}$ in coronal sections), with elongated or triangular cell bodies and more abundant cytoplasm (Fig. 5A, arrow). These features of the TLC contrast with the ventrally adjacent PAGdm, whose neurons

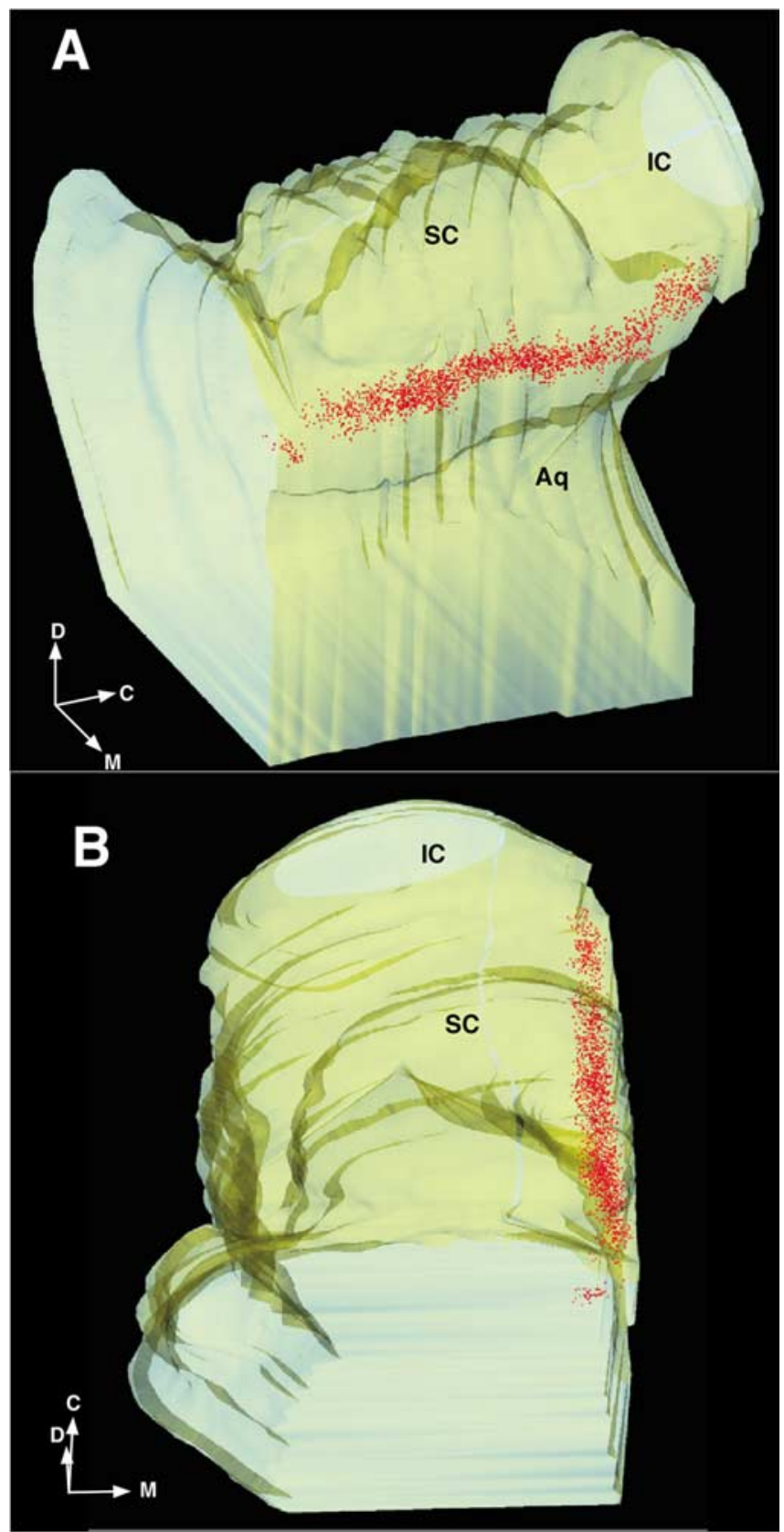

Figure 4. Three-dimensional reconstruction of the rat TLC. Three-dimensional reconstruction of the position of TLC neurons retrogradely labeled after an injection of FluoroGold into the right superior olivary complex. To favor the observation of the labeled neurons, the left side of the mesencephalon has been removed and the right side is depicted as a semitransparent, yellow solid structure. Each red dot represents one TLC labeled neuron. To provide a sharper, cleaner image, the 3D reconstruction was based on every other section; therefore, the actual number of labeled neurons was considerably higher than the number of dots. When all of the sections are used for the 3D reconstruction, the seemingly isolated group of neurons in the rostral end of the TLC is clearly identified as part of the nucleus. $A$, View from a point located in the left side and slightly elevated. $\boldsymbol{B}$, View from a point located rostral and dorsal. The vision angle is roughly parallel to the midline and forms a $45^{\circ}$ angle with the horizontal plane. Many more neurons were labeled in the central third of the TLC than in the caudal rostral thirds. M, Medial; C, caudal; D, dorsal; Aq, cerebral aqueduct.

show greater heterogeneity, larger average size ( $t$ test, $p<0.0001$; TLC, $n=132$; PAGdm, $n=175$ ), darker Nissl staining, lower packing density ( $t$ test, $p<0.0001$; TLC, $n=100$; PAGdm, $n=$ $100 ; n$ here refers to number of squares where cells were counted) (see Materials and Methods) and random orientation of the cell 

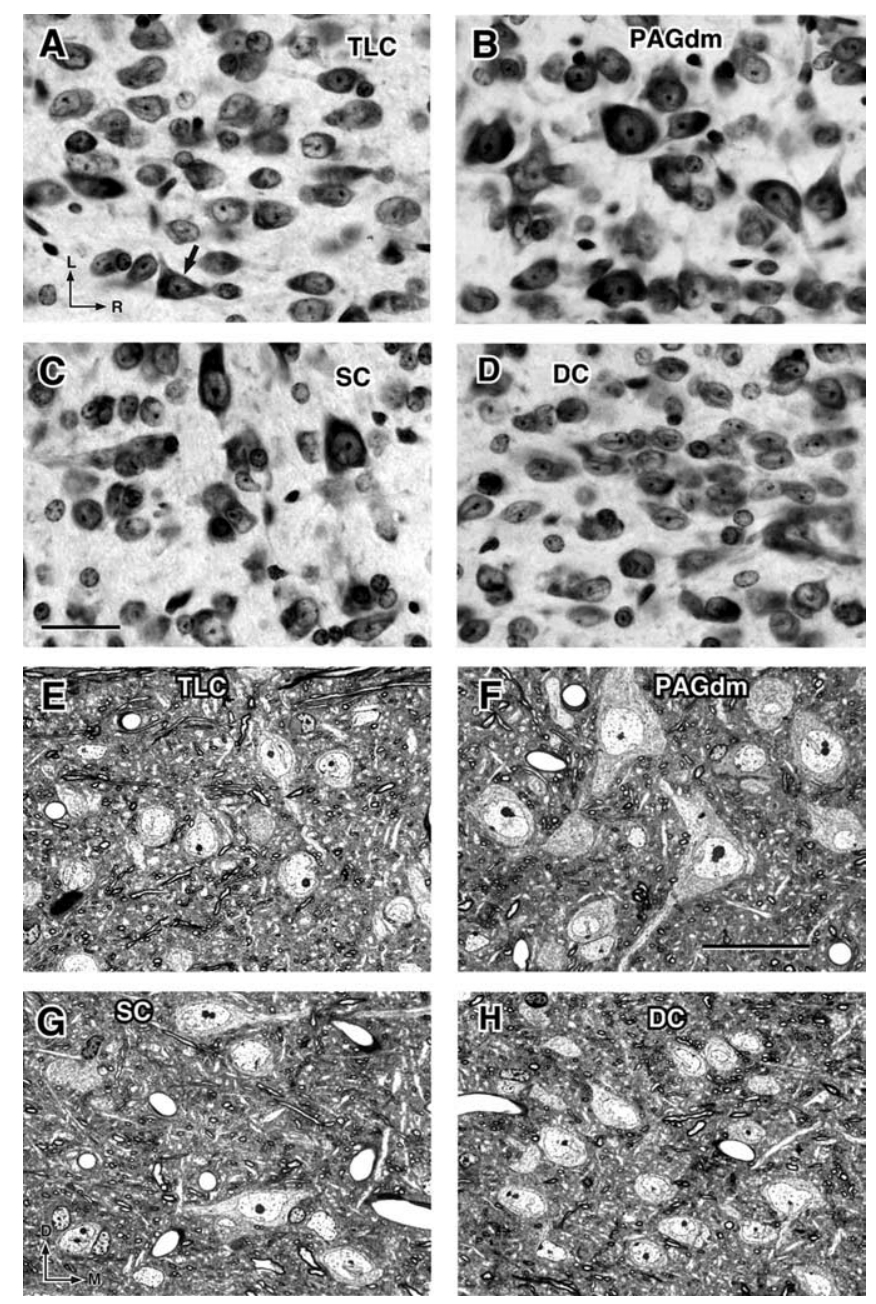

Figure 5. Cytoarchitectural features of the rat TLC. $\boldsymbol{A}-\boldsymbol{D}$, Digital micrographs of $15 \mu \mathrm{m}$ thick, paraffin embedded, horizontal sections of the rat midbrain tectum stained by the Nissl method. They illustrate the typical cytoarchitectural features of the $\operatorname{TLC}(\boldsymbol{A}), \operatorname{PAGdm}(\boldsymbol{B})$, medial SC $(C)$, and dorsal column (DC) (D). The arrow in $A$ indicates a typical medium-sized neuron. All four microscopic fields come from a similar rostrocaudal level that corresponds to the central third of the TLC. Scale bar: $25 \mu \mathrm{m}$, uncorrected for shrinkage. $\boldsymbol{E}-\boldsymbol{H}$, High-magnification digital micrographs taken from a single semithin, coronal section through the central third of the TLC. They illustrate the typical cytoarchitectural features of the TLC $(\boldsymbol{E})$, PAGdm $(\boldsymbol{F})$, medial SC (G), and dorsal column $(\boldsymbol{H})$. Toluidine blue stain. Scale bar, $25 \mu \mathrm{m}$.

bodies (Fig. $5 B, F$ ). TLC neurons also contrast with the laterally adjacent deep SC, whose neurons are also larger $(t$ test, $p<$ 0.0001; TLC, $n=132$; SC, $n=185)$, less densely packed ( $t$ test, $p<0.0001$; TLC, $n=100$; SC, $n=100$ ), and more heterogeneous in size, shape and orientation (Fig. $5 C, G$ ). No TLC neuron is as large as the large neurons in the PAGdm, the medial deep SC or the medial IC. Notably, IC neurons tend to be more triangular or multipolar than TLC neurons.

Dorsal to the TLC is a distinct territory characterized by a homogeneous population of small neurons whose orientation and cytological features resemble at first glance those of TLC small neurons (Figs. $1 G-I, 5 D, H$ ). This dorsal territory may constitute another distinct and previously unnoticed columnar nucleus that parallels the TLC and whose further characterization awaits future studies. We will refer to this neuronal assembly dorsal to the TLC as the dorsal column. The dorsal column can be distinguished from the TLC because: (1) its neuronal somata are slightly, but significantly smaller than those of TLC small neurons ( $t$ test, $p<0.0001$; TLC, $n=111$; dorsal column, $n=122$ ), (2) it
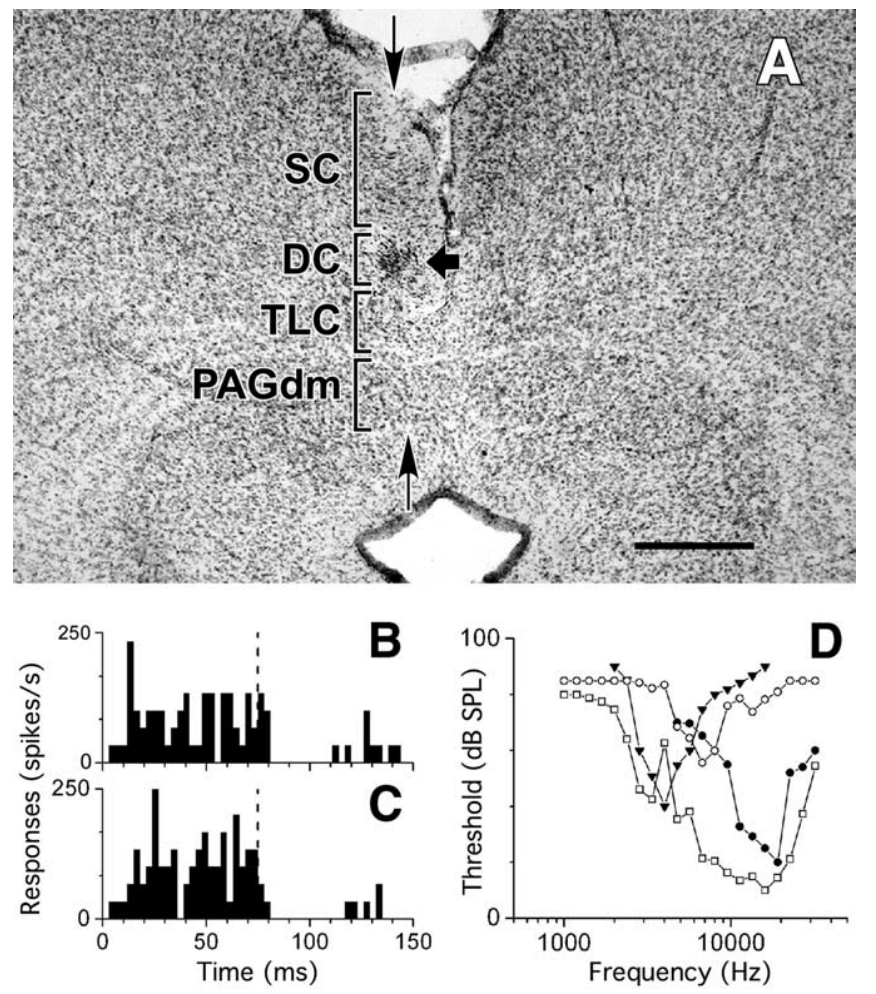

Figure 6. Responses of TLC neurons to acoustic stimuli. A, Digital micrograph of a coronal section of the rat midbrain tectum with a visible electrode tract. The dorsal and ventral ends of the tract are indicated by the vertical arrows. As the electrode advanced dorsoventrally, it successively crossed the medial superficial SC, the dorsal column (DC), the TLC, and the PAGdm, whose limits are indicated by brackets. The thick arrow points to an electrolytic lesion made in the $D C$ as the electrode was withdrawn past the auditory responsive TLC. Scale bar, $1 \mathrm{~mm}$. $\boldsymbol{B}$, Poststimulus time histogram of the response of a single neuron to the auditory search stimulus (wideband noise, $0-12000 \mathrm{~Hz}$ at $80 \mathrm{dBSPL}$ ) applied to both ears. The end of the stimulus period (75 ms) is indicated by the dashed line. C, Poststimulus time histogram of the response of the same neuron as in $\boldsymbol{B}$ to a tone at best frequency ( $18.5 \mathrm{kHz}$ at $70 \mathrm{~dB} \mathrm{SPL}$ ) applied to both ears. The end of the stimulus period (75 ms) is indicated by the dashed line. $\boldsymbol{D}$, Representative tuning curves from single neurons in the TLC. A mix of neurons with broad and narrow tuning was seen.

lacks for the most part neurons comparable with TLC mediumsized neurons, (3) its neuronal packing density is significantly higher than that of the TLC ( $t$ test, $p<0.0001$; TLC, $n=100$; dorsal column, $n=100$ ), (4) it is not traversed by fascicles of the CoIC or the CoSC, (5) its neurons are not labeled after injections of FluoroGold into the superior olivary complex (Fig. 2), and (6) as explained below, its electrophysiological features differ from those of the TLC.

\section{The TLC is electrophysiologically distinct}

Because TLC neurons are labeled after injections of retrograde tracers into the superior olivary complex (Faye-Lund, 1986; present study), the TLC was examined physiologically to determine whether its neurons were responsive to sound. As albino and pigmented rats are known to differ in various aspects of the structure and physiology of their auditory systems (for review, see Grant and Binns, 2003), we recorded from single neurons and small clusters of neurons $(n=87)$ in the TLC of Sprague Dawley and Long-Evans rats, with similar results for both strains. After traversing the cerebral cortex, a typical vertical electrode penetration (Fig. 6A) encountered light-sensitive neuronal responses in the SC. No auditory responsiveness was encountered in this extremely medial part of the superficial SC. This light-sensitive region was followed by a 200 - to $300-\mu \mathrm{m}$-wide gap with no neural 
responses to sound or light, which presumably corresponded to the aforementioned dorsal column. The electrode then entered the TLC, marked by a clear and often robust background of auditory responsiveness, and no sensitivity to light. Past the TLC, the electrode entered the PAGdm, where neurons did not respond to acoustic stimuli. Figure $6 B$ shows a representative example of the responses of a single TLC neuron to the auditory search stimulus (wideband noise, 0-12000 Hz), and a comparable response to tones at the unit's best frequency $(18.5 \mathrm{kHz})$ is shown in Figure 6C. These responses were sustained, which was the most common response in TLC neurons. The sharpness of frequency tuning and thresholds varied among TLC neurons, as shown by a few representative tuning curves from single neurons (Fig. 6D). Across neurons, the first spike latencies tended to be long (median of $18.6 \mathrm{~ms}$, semi-interquartile range of $5.97 \mathrm{~ms}$ ).

These results indicate that TLC neurons are indeed responsive to acoustic stimuli. A comparison with data from the literature reveals that the responses of TLC neurons to sound are different from those of SC neurons. All TLC neurons in our sample responded to sound, but not to static visual stimuli, whereas $21 \%$ of the auditory-responsive neurons in the rat SC also respond to static visual stimuli (Gaese and Johnen, 2000). Moreover, the characteristic frequencies of TLC neurons were widely distributed within the hearing range of the animal, and this finding differs from the predominance of neurons with high characteristic frequency in the deep SC of the cat (Wise and Irvine, 1983; Hirsch et al., 1985). Finally, the sustained responses to sound in most TLC neurons are uncommon among auditory-responsive neurons in the cat SC (Hirsch et al., 1985).

The responses of TLC neurons differ also from those of rat IC neurons. First, although our responses were sampled throughout the rostrocaudal extent of the TLC, no tonotopic organization was evident; this observation clearly contrasts with the well known tonotopic map of the IC (Kelly et al., 1991). Moreover, tuning curves and frequency response areas were in general somewhat wider in the TLC than in the IC (Kelly et al., 1991; Hernández et al., 2005), which indicates that TLC neurons are less selective to sound frequency. This is particularly true for neurons sensitive to high frequency sounds, whose outstanding frequency selectivity in the IC (Kelly et al., 1991; Hernández et al., 2005) was not matched by the corresponding neurons in the TLC. Furthermore, sustained responses were more common in the TLC than in the IC. Finally, the median response latencies were considerably longer in the TLC than in the IC (Kelly et al., 1991).

\section{The TLC is present in a large variety of mammals}

To determine whether the existence of the TLC is species specific or is evolutionarily conserved, we examined histological sections of the midbrain tectum of various mammalian species frequently used as experimental animals, as well as sections of human brainstems. Without exception, the TLC was readily distinguished from the neighboring nuclei by a host of cytoarchitectural criteria (Nieuwenhuys, 1998), including neuronal density and the shape, size, orientation and staining pattern of its cell bodies. By morphologic criteria, the identification of the TLC in the mesencephalon of Rattus norvegicus was extended to mouse (Mus musculus), gerbil (Meriones unguiculatus), hamster (Mesocricetus auratus), chinchilla (Chinchilla lanigera), guinea pig (Cavia porcellus), rabbit (Oryctolagus cuniculus), cat (Felis catus), ferret (Mustela putorius), crab-eating monkey (Macaca fascicularis), and human (Homo sapiens sapiens). Figure 7 shows representative micrographs of the TLC in some of these species.

\section{Discussion}

The TLC: a distinct nucleus of the mammalian brain

Our data derived from studies of fiber connections, cytoarchitecture and electrophysiologic recordings demonstrate that the TLC is a distinct nucleus of the rat brain. It has connections with auditory centers and its neurons are responsive to auditory stimuli. Despite its narrow appearance in coronal sections, the size of this longitudinally oriented structure is impressive: in the rat its length $(3.5 \mathrm{~mm})$ represents almost $20 \%$ of the entire cerebral length, and the nucleus contains as many neurons, or more, than other auditory centers of the brainstem, including many nuclei of 
the superior olivary complex and lateral lemniscus (Kulesza et al., 2002).

It may seem surprising that a nucleus as large as the TLC has remained unnoticed until now. Several factors, some of them technical, may have contributed to this neglect. First, the midsagittal plane is covered by the superior sagittal sinus, a vessel whose large diameter and abundant blood flow may act as deterrent for numerous experiments. Second, the cytoarchitecture of the TLC, although distinct at close inspection, shares some similarities with the medial border of the SC, which explains why the territory occupied by the rostral twothirds of the TLC has been traditionally considered the most medial part of the deep SC (Olszewski and Baxter, 1954; Berman, 1968; Swanson, 1999; Hof et al., 2000; Paxinos and Watson, 2005). Third, with respect to the caudal third of the TLC, located within the IC, previous authors have mentioned a "commissural nucleus" or "interstitial nucleus" between the midline and the medial border of the dorsal cortex of the IC. However, the commissural region identified by these authors includes, but is not limited to, the caudal third of the TLC, and its continuity with the rostral two-thirds of the TLC was not in general noted (Geniec and Morest, 1971; Willard and Ryugo, 1983; Morest and Oliver, 1984; Faye-Lund and Osen, 1985; Herrera et al., 1987, 1988a,b). Finally, another reason that the TLC may have gone unnoticed is that many commonly used neurochemical markers do not highlight the nucleus. For example, in sections of the rat midbrain stained for parvalbumin, calbindin, calretinin, substance $\mathrm{P}$, calcitonin generelated peptide, neurofilament protein (SMI-32), tyrosine hydroxylase, NADPH-diaphorase, acetylcholinesterase, or adenosine deaminase, the TLC is hardly distinguishable (Miguel-Hidalgo et al., 1989; Paxinos et al., 1999).

Some reports in the literature did identify important features of the TLC, adding strength to its present characterization. After injection of a retrograde tracer in the superior olivary complex, Faye-Lund (1986) reported "a distinct group of labeled cells in the commissure of the IC, close to the midline on the application side. Rostrally the cell group continued into the medialmost part of the superior colliculus." Saldaña and Merchán (1992) reported that some of the fibers of the rat IC that traveled in the CoIC gave off collaterals that created two terminal fields, one on each side of the midline, which extended rostrally and reached the level of the SC [Saldaña and Merchán (1992), their Figs. 4A-D, 7A-D]. Shortly thereafter, Saldaña et al. (1996) noticed terminal fields in the same location formed by collaterals of auditory corticocollicular fibers that crossed the CoIC [Saldaña et al. (1996), their Fig. 9B,C]. Furthermore, a continuous pool of neurons expressing high levels of glutamic acid decarboxylase, the synthesizing enzyme for GABA, was reported at the CoIC and the CoSC of the rat (Mugnaini and Oertel, 1985). Together, these findings support a role for the TLC in auditory function and the extent of the TLC described in the present study.

In conclusion, the organization of the TLC is clearly different from the concentric fibrocellular laminas of the IC (Saldaña and Merchán, 2005) and from the alternating horizontal layers of gray and white matter of the SC (May, 2005). It also differs from the mosaic-like pattern of vertical patches and modules found throughout the SC (Chevalier and Mana, 2000; Harting, 2004). Therefore, the discovery of the TLC reveals unexpected levels of longitudinal organization in the mammalian midbrain tectum. Interestingly, the longitudinal axis of the TLC is in register with the axis of the functional columns of the PAG (Behbehani, 1995; Ruiz-Torner et al., 2001). Although the close apposition between the TLC and the PAGdm (Beitz and Shepard, 1985; Herrera et al., 1988b) raises interesting functional questions, we do not consider the TLC as a dorsal extension of the PAG, because these two structures differ markedly in their cytoarchitecture, relationship with the tectal commissures, connections and electrophysiology.

\section{What is the role of the TLC?}

Despite the limited information currently available about the TLC, our results provide a conceptual framework for future studies. The fact that the TLC is found in species as different as rodents, lagomorphs, carnivores and primates suggests that it may be common to all terrestrial mammals, or even to the entire mammalian class. The function of the TLC is, therefore, probably shared by a large variety of animals.

Insight into the biological significance of the TLC can be gained from its physiology and connections. In the rat, the TLC is innervated by the IC (Saldaña and Merchán, 1992) and by the auditory neocortex (Saldaña et al., 1996), and similar connectivity is apparent in the cat (Morest and Oliver, 1984). Moreover, the TLC is crossed by the CoIC, which includes projections from yet additional auditory structures, including the superior paraolivary nucleus, the sagulum and the nuclei of the lateral lemniscus (Saldaña and Merchán, 2005). These putative connections with multiple auditory centers, combined with responsiveness to auditory stimuli demonstrated here, suggest that TLC neurons are involved in the processing of acoustic information. The long latencies with which most TLC neurons respond to sound indicate that the nucleus is probably not part of the core ascending auditory pathway, but likely serves a role in descending auditory pathways. This suggestion is supported by the tract-tracing experiments, which show that TLC neurons project to the ipsilateral superior olivary complex.

Additional studies are needed to determine whether the TLC is involved in multisensory and/or sensorimotor integration, a role that would be consistent with its tectal location. Previous studies have shown that certain auditory nuclei that were previously considered predominantly unimodal play a role in multisensory processing (Bulkin and Groh, 2006). Moreover, the TLC is crossed by the CoSC, a complex tract that contains axons from over forty tectal and nontectal sources (Huerta and Harting, 1984) and whose contribution to the innervation of the TLC remains unknown. It will be particularly interesting to establish whether TLC neurons receive direct input from commissural SC neurons, most of which participate in the processing of visual information (McIlwain, 1991). The fact that the crossed projections of the SC innervate the most medial regions of the SC (Yamasaki et al., 1984; Rhoades et al., 1986; Behan and Kime, 1996; Chebat et al., 2006) lends support to this possibility. Extending our knowledge of the auditory properties of TLC neurons, determining their responses to light and other sensory modalities, and unraveling the afferent and efferent connections of the nucleus will lead to a much better understanding of its functional significance.

The TLC presents itself as an unexpected and promising focus for future research on mammalian auditory function. Knowledge about the nucleus is still rudimentary but, with currently available technologies, neuroscientists should be able to accomplish in a few years what took many decades to achieve in other nuclei of the brain. 


\section{References}

Aitkin L (1986) The auditory midbrain: structure and function in the central auditory pathway. Clifton, NJ: Humana.

Behan M, Kime NM (1996) Spatial distribution of tectotectal connections in the cat. Prog Brain Res 112:131-142.

Behbehani MM (1995) Functional characteristics of the midbrain periaqueductal gray. Prog Neurobiol 46:575-605.

Beitz AJ, Shepard RD (1985) The midbrain periaqueductal gray in the rat. II. A Golgi analysis. J Comp Neurol 237:460-475.

Berman AL (1968) The brain stem of the cat: a cytoarchitectonic atlas with stereotaxic coordinates. Madison, WI: University of Wisconsin.

Bulkin DA, Groh JM (2006) Seeing sounds: visual and auditory interactions in the brain. Curr Opin Neurobiol 16:415-419.

Chebat DR, Boire D, Ptito M (2006) Development of the commissure of the superior colliculus in the hamster. J Comp Neurol 494:887-902.

Chevalier G, Mana S (2000) Honeycomb-like structure of the intermediate layers of the rat superior colliculus, with additional observations in several other mammals: AChE patterning. J Comp Neurol 419:137-153.

Edwards SB (1977) The commissural projection of the superior colliculus in the cat. J Comp Neurol 173:23-40.

Faye-Lund H (1986) Projection from the inferior colliculus to the superior olivary complex in the albino rat. Anat Embryol (Berl) 175:35-52.

Faye-Lund H, Osen KK (1985) Anatomy of the inferior colliculus in rat. Anat Embryol (Berl) 171:1-20.

Gaese BH, Johnen A (2000) Coding for auditory space in the superior colliculus of the rat. Eur J Neurosci 12:1739-1752.

Geniec P, Morest DK (1971) The neuronal architecture of the human posterior colliculus. A study with the Golgi method. Acta Otolaryngol [Suppl] 295:1-33.

Grant S, Binns KE (2003) Reduced influence of the ipsilateral ear on spatial tuning of auditory neurons in the albino superior colliculus: a knock-on effect of anomalies of the acoustic chiasm? Exp Brain Res 151:478-488.

Gundersen HJ (1988) The nucleator. J Microsc 151:3-21.

Gundersen HJ, Bagger P, Bendtsen TF, Evans SM, Korbo L, Marcussen N, Møller A, Nielsen K, Nyengaard JR, Pakkenberg B, Sørensen FB, Vesterby A, West MJ (1988) The new stereological tools: disector, fractionator, nucleator and point sampled intercepts and their use in pathological research and diagnosis. APMIS 96:857-881.

Hall WV, Moschovakis A (2004) The superior colliculus: new approaches for studying sensorimotor integration. Boca Raton, FL: CRC.

Harting JK (2004) Puffs and patches: a brief chronological review. In: The superior colliculus: new approaches for studying sensorimotor integration (Hall WV, Moschovakis A, eds), pp 83-105. Boca Raton, FL: CRC.

Hernández O, Espinosa N, Pérez-González D, Malmierca MS (2005) The inferior colliculus of the rat: a quantitative analysis of monaural frequency response areas. Neuroscience 132:203-217.

Herrera M, Sánchez del Campo F, Smith Agreda V (1987) The commissural nucleus of the inferior colliculus in the rabbit. A morphological study. J Hirnforsch 28:671-683.

Herrera M, Sánchez del Campo F, Puchades Orts A (1988a) Cytoarchitecture and axonal systems in the commissural nucleus of the inferior colliculus in the albino rat. A Golgi study. J Hirnforsch 29:165-174.

Herrera M, Sánchez del Campo F, Ruiz A, Smith Agreda V (1988b) Neuronal relationships between the dorsal periaqueductal nucleus and the inferior colliculus (nucleus commissuralis) in the cat. A Golgi study. J Anat 158:137-145.

Hirsch JA, Chan JC, Yin TCT (1985) Responses of neurons in the cat's superior colliculus to acoustic stimuli. I. Monaural and binaural response properties. J Neurophysiol 53:726-745.

Hof PR, Young WG, Bloom FE, Belichenko PV, Celio MR (2000) Comparative cytoarchitectonic atlas of the C57BL/6 and 129/Sv mouse brains. Amsterdam: Elsevier.

Huerta MF, Harting JK (1984) The mammalian superior colliculus: studies of its morphology and connections. In: Comparative neurology of the optic tectum (Vanegas H, ed), pp 687-773. New York: Plenum.

Íñiguez C, Gayoso MJ, Carreres J (1985) A versatile and simple method for staining nervous tissue using Giemsa dye. J Neurosci Methods 13:77-86.

Kelly JB, Glenn SL, Beaver CJ (1991) Sound frequency and binaural re- sponse properties of single neurons in rat inferior colliculus. Hear Res 56:273-280.

King AJ (2004) The superior colliculus. Curr Biol 14:R335-R338.

Kulesza RJ, Viñuela A, Saldaña E, Berrebi AS (2002) Unbiased stereological estimates of neuron number in subcortical auditory nuclei of the rat. Hear Res 168:12-24.

Leergaard TB, Bjaalie JG (1995) Semi-automatic data acquisition for quantitative neuroanatomy. MicroTrace-computer programme for recording of the spatial distribution of neuronal populations. Neurosci Res 22:231-243.

May PJ (2005) The mammalian superior colliculus: laminar structure and connections. Prog Brain Res 151:321-378.

McIlwain JT (1991) Visual input to commissural neurons of the cat's superior colliculus. Vis Neurosci 7:389-393.

Miguel-Hidalgo JJ, Senba E, Matsutani S, Takatsuji K, Fukui H, Tohyama M (1989) Laminar and segregated distribution of immunoreactivities for some neuropeptides and adenosine deaminase in the superior colliculus of the rat. J Comp Neurol 280:410-423.

Morest DK, Oliver DL (1984) The neuronal architecture of the inferior colliculus in the cat: defining the functional anatomy of the auditory midbrain. J Comp Neurol 222:209-236.

Mugnaini E, Oertel WH (1985) An atlas of the distribution of GABAergic neurons and terminals in the rat CNS as revealed by GAD immunocytochemistry. In: Handbook of chemical neuroanatomy, Vol IV, GABA and neuropeptides in the CNS, Pt I (Björlund A, Hökfelt T, eds), pp 436-608. Amsterdam: Elsevier.

Nieuwenhuys R (1998) Structure and organization of centres. In: The central nervous system of vertebrates (Nieuwenhuys R, Ten Donkelaar HJ, Nicholson C, eds), pp 113-157. Berlin: Springer.

Olivier E, Corvisier J, Pauluis Q, Hardy O (2000) Evidence for glutamatergic tectotectal neurons in the cat superior colliculus: a comparison with GABAergic tectotectal neurons. Eur J Neurosci 12:2354-2366.

Olszewski J, Baxter D (1954) Cytoarchitecture of the human brainstem. Philadelphia: Lippincott, Williams and Wilkins.

Paxinos G, Watson C (2005) The rat brain in stereotaxic coordinates, Ed 5. San Diego: Elsevier-Academic.

Paxinos G, Kus L, Ashwell K, Watson C (1999) Chemoarchitectonic atlas of the rat forebrain. San Diego: Elsevier-Academic.

Rhoades RW, Mooney RD, Szczepanik AM, Klein BG (1986) Structural and functional characteristics of commissural neurons in the superior colliculus of the hamster. J Comp Neurol 253:197-215.

Ruiz-Torner A, Olucha-Bordonau F, Valverde-Navarro AA, MartínezSoriano F (2001) The chemical architecture of the rat's periaqueductal gray based on acetylcholinesterase histochemistry: a quantitative and qualitative study. J Chem Neuroanat 21:295-312.

Saldaña E, Merchán MA (1992) Intrinsic and commissural connections of the rat inferior colliculus. J Comp Neurol 319:417-437.

Saldaña E, Merchán MA (2005) Intrinsic and commissural connections of the inferior colliculus. In: The inferior colliculus (Winer JA, Schreiner CE, eds), pp 155-181. New York: Springer.

Saldaña E, Feliciano M, Mugnaini E (1996) Distribution of descending projections from primary auditory neocortex to inferior colliculus mimics the topography of intracollicular projections. J Comp Neurol 371:15-40.

Sparks DL, Nelson IL (1987) Sensory and motor maps in the mammalian superior colliculus. Trends Neurosci 10:312-317.

Swanson LW (1999) Brain maps: structure of the rat brain, Ed 2. New York: Elsevier.

Thompson AM, Schofield BR (2000) Afferent projections of the superior olivary complex. Microsc Res Tech 51:330-354.

Willard FH, Ryugo DK (1983) Anatomy of the central auditory system. In: The auditory psychobiology of the mouse (Willot JF, ed), pp 201-304. Springfield, IL: Charles C. Thomas.

Winer JA, Schreiner CE (2005) The inferior colliculus. New York: Springer Science and Business Media.

Wise LZ, Irvine DR (1983) Auditory response properties of neurons in deep layers of cat superior colliculus. J Neurophysiol 49:674-685.

Yamasaki DS, Krauthamer G, Rhoades RW (1984) Organization of the intercollicular pathway in rat. Brain Res 300:368-371. 